\title{
Band structure engineering of two-dimensional magnonic vortex crystals
}

\author{
Carolin Behncke,,${ }^{1, *}$ Max Hänze, ${ }^{1}$ Christian F. Adolff, ${ }^{1}$ Markus Weigand, ${ }^{2}$ and Guido Meier ${ }^{3,4}$ \\ ${ }^{1}$ Institut für Angewandte Physik und Zentrum für Mikrostrukturforschung, Universität Hamburg, Jungiusstr. 11, 20355 Hamburg, Germany \\ ${ }^{2}$ Max-Planck Institute for Intelligent Systems, Heisenbergstr. 3, 70569 Stuttgart, Germany \\ ${ }^{3}$ Max-Planck Institute for the Structure and Dynamics of Matter, Luruper Chaussee 149, 22761 Hamburg, Germany \\ ${ }^{4}$ The Hamburg Centre for Ultrafast Imaging, Luruper Chaussee 149, 22761 Hamburg, Germany \\ (Received 20 February 2015; revised manuscript received 20 May 2015; published 15 June 2015)
}

\begin{abstract}
Magnonic vortex crystals are studied via scanning transmission x-ray microscopy and ferromagnetic-resonance spectroscopy. We investigate a two-dimensional vortex crystal by imprinting waves with tunable wave vectors. The dispersion relation $\omega(k)$ is determined via ferromagnetic-resonance spectroscopy with a tunable frequency and wave vector for two vortex core polarization patterns that are adjusted by self-organized state formation prior to the measurement. We demonstrate that the band structure of the crystal is reprogrammed by tuning the vortex polarizations.
\end{abstract}

DOI: 10.1103/PhysRevB.91.224417

PACS number(s): 75.70.Kw, 71.20.-b, 68.37.Yz, 76.50.+g

\section{INTRODUCTION}

The concept of phononic and electronic band structures in crystalline solids that are based on ordered arrangements of interacting elements is one of the major accomplishments of solid state physics. Engineering of the band structure led to important applications, such as the blue light-emitting diode [1,2]. The properties of artificial crystals can be tuned at will, paving the way to customizable band structures in electronic, photonic, and magnonic systems. A periodic arrangement of ferromagnetic nanodisks in the vortex state can be described as an artificial crystal. In analogy to photonic crystals, it is called a magnonic crystal $[3,4]$ and is expected to feature a group velocity, a density of states, and a band structure.

The magnetic vortex state forms in ferromagnetic nanodisks of suitable geometry. The magnetization curls in the plane around the vortex core in the center region where the magnetization points out-of-plane [5]. The vortex is described by two state parameters: the polarization of the magnetization in the core pointing either up or down $(p= \pm 1)$ and the chirality, the sense of the in-plane magnetization curling either clockwise or counterclockwise $(C= \pm 1)$ [6]. The vortex core can be resonantly excited by magnetic fields and electric currents $[7,8]$. This leads to a high frequency gyrotropic motion of the vortex around its center position $[9,10]$. Depending on the polarization the sense of gyration is clockwise or counterclockwise [7]. When deflected from their equilibrium position vortices couple via their rotating magnetic stray fields [11]. The collective motions of an arrangement of vortices can be understood by the oscillations of dipolar coupled vortices as building blocks. Due to the different relative motions, the coupling strength depends on the relative polarization orientation of the vortices [12]. Thus, the properties of the artificial crystal cannot only be tailored by the geometry of the crystal but also by tuning the polarization configuration. Experimentally, the interaction between the vortices in twodimensional arrangements has been studied [13-16] and

\footnotetext{
*cbehncke@physnet.uni-hamburg.de
}

collective modes for five vortices have been measured [17]. The band structure of two-dimensional vortex crystals is predicted to strongly depend on the polarization pattern [18-20].

In this paper we show experimentally that the band structure of a vortex crystal can be modified via the polarization patterns. The polarization configuration is tuned via self-organized state formation, see Ref. [13]. We use a specific setup of ferromagnetic-resonance spectroscopy (FMR) to observe the collective behavior of the crystal in the frequency domain [Fig. 1(a)]. Scanning transmission X-ray microscopy (STXM) at the MAXYMUS microscope of the BESSY II synchrotron in Berlin, Germany, is used to observe the vortex motions temporally and spatially resolved. The measurements are substantiated and combined to a conclusive picture with calculations based on an extended Thiele model $[15,21]$.

\section{SAMPLES}

Permalloy $\left(\mathrm{Ni}_{80} \mathrm{Fe}_{20}\right)$ disks depicted in Fig. 1(b) were prepared by electron-beam lithography, $\mathrm{O}_{2}$ plasma etching, thermal evaporation, and lift-off processing on $100 \mathrm{~nm}$ thick silicon nitride membranes transparent for soft $\mathrm{x}$ rays. Two different sample types were prepared in order to adapt to the assets of the two measurement techniques. Sample type I is used to characterize the polarization pattern tuning with STXM, and sample type II is optimized for band structure measurements with FMR. Samples of type I consist of $50 \times 50$ disks that are $t=40 \mathrm{~nm}$ thick, have a radius of $R=500 \mathrm{~nm}$, and a center-to-center distance of $D=1.15 \mu \mathrm{m}$. For sample type II $25 \times 25$ disks were deposited with the spatial dimensions $t=60 \mathrm{~nm}, R=1 \mu \mathrm{m}$, and $D=2.05 \mu \mathrm{m}$. Striplines of copper with a thickness of $120 \mathrm{~nm}$ and an oxidation protection layer of $5 \mathrm{~nm}$ of gold were deposited on top of the disks. A high frequency signal driven through the striplines leads to an alternating magnetic field $\vec{H}_{\mathrm{rf}}=\left(H_{0} \sin (\omega t), 0,0\right)$ in the $x$ direction [Fig. 1(a)] and excites the gyrotropic motion of the vortices. The small disks of type I are beneficial to observe many disks in the field of view of the $\mathrm{x}$-ray microscope. The enlarged distance between the two thin striplines in sample type II reduces the cross talk between the striplines and improves the electrical signal used for FMR spectroscopy. 

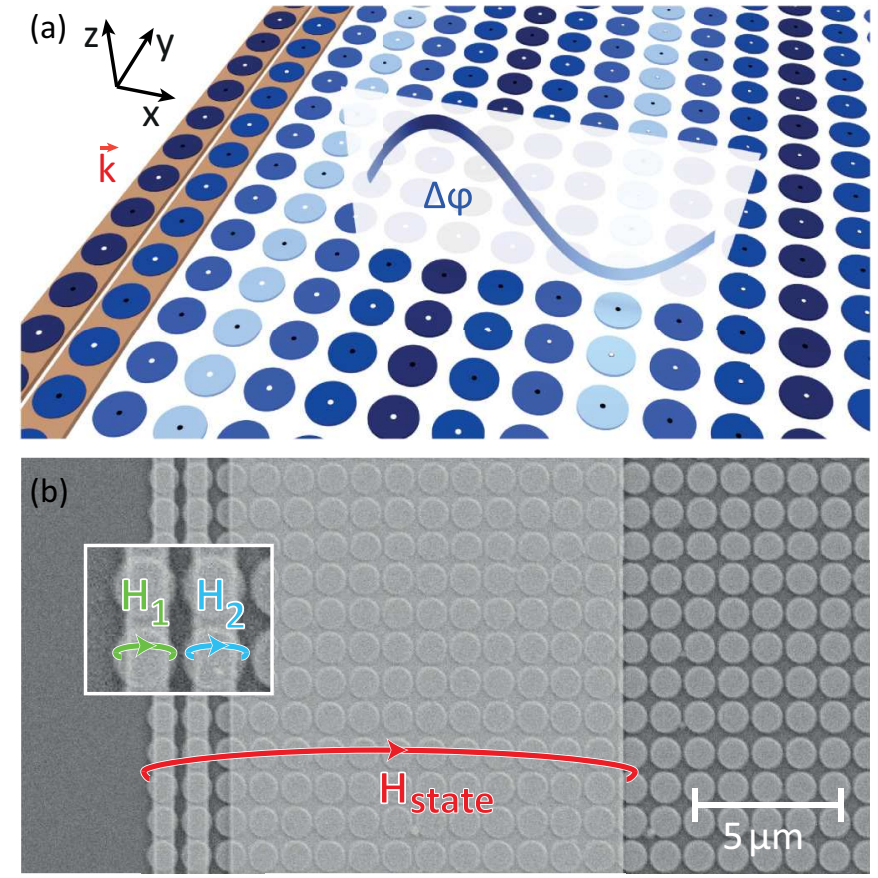

FIG. 1. (Color online) (a) Schematic representation of gyration waves propagating through a two-dimensional vortex crystal imprinted by a phase-shifted excitation of the first two columns of vortices. (b) Scanning electron micrograph of a vortex crystal covered by copper striplines. All three striplines are used to tune the polarization pattern by self-organized state formation. The two thin striplines are used for band structure measurements.

\section{STATE FORMATION IN VORTEX CRYSTALS}

The polarization pattern of the vortex crystal is tuned by self-organized state formation $[13,16,22]$. Therefore a high frequency current is applied to all three striplines [Fig. 1(b)] that results in a magnetic Oersted field of maximal $\mu_{0} H_{\text {state }}=1.26 \mathrm{mT}$ and is adiabatically reduced on a time scale of milliseconds. The STXM measurements in Fig. 2(a) reveal the polarization pattern of the vortices under the broad stripline of sample type I subsequent to the state formation at the frequencies $f_{\text {state }}=270,320$, and $330 \mathrm{MHz}$ marked in Fig. 2(b). In order to determine the polarization state, the vortices are excited by a small alternating high frequency field of $\mu_{0} H_{0}=0.09 \mathrm{mT}$ that does not affect the polarization configuration. In the disks covered by the striplines the polarization pattern is reliably tuned. Differences might occur in the disks not covered by the striplines. The size of the black or white dots in Fig. 2(a) represents the relative gyration amplitude during this noninvasive excitation. We observe a horizontally striped polarization pattern at $f_{\text {state }}=270 \mathrm{MHz}$, while for state formation at 320 or $330 \mathrm{MHz}$ large domains of homogeneous polarization emerge (see movie 1 in the Supplemental Material [23]). The tiny black dots in the white domain of the polarization pattern for $f_{\text {state }}=320 \mathrm{MHz}$ exhibit a small gyration amplitude and cannot be determined properly [24]. The size of the domains, i.e., the number of vortices in an equally polarized region, after the state formation at $330 \mathrm{MHz}$ is decreased in comparison to a state formation frequency of $320 \mathrm{MHz}$. With increasing state formation frequency the
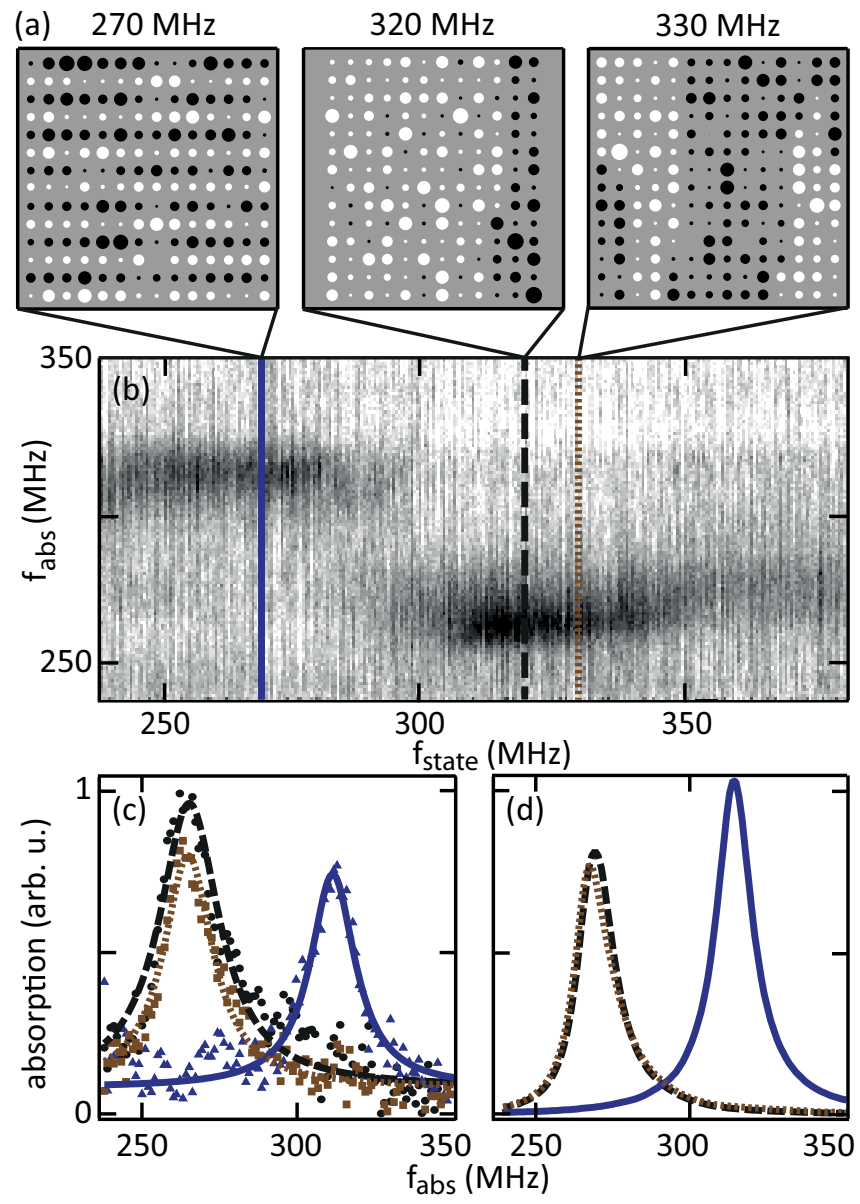

FIG. 2. (Color online) (a) STXM measurements after state formation with a frequency $f_{\text {state }}=270 \mathrm{MHz}, 320 \mathrm{MHz}$, and $330 \mathrm{MHz}$. The white and black dots correspond to $p=1$ and $p=-1$, respectively. Their size depicts the relative gyration amplitude. (b) Absorption spectra of five $50 \times 50$ vortex arrays after state formation with varying frequencies measured with the broad stripline shown in Fig. 1(b). (c) Selected absorption spectra after state formation at $270 \mathrm{MHz}$ (solid dark blue), $320 \mathrm{MHz}$ (dashed black), and $330 \mathrm{MHz}$ (dotted brown) and Lorentzian fit curves. (d) Absorption spectra calculated within the extended Thiele model corresponding to the following polarization patterns: horizontally striped polarization (solid dark blue), big domains (dashed black), and smaller domains (dotted brown).

pattern approaches the vertically striped pattern, while with decreasing state formation frequency the pattern approaches the homogeneous polarization pattern. For frequencies below $290 \mathrm{MHz}$ the horizontally striped pattern is tuned. Thus, by changing the state formation frequency different polarization patterns are tuned on purpose. This behavior can also be measured in the frequency domain. Figure 2(b) shows the absorption spectra of FMR measurements at different state formation frequencies where black contrast corresponds to strong absorption. The vortex crystal is uniformly excited via the broad stripline [Fig. 1(b)] by a sinusoidal magnetic field with different frequencies $f_{\text {abs }}$ that does not affect the polarization configuration. The absorption is determined with the help of a reference measurement at $60 \mathrm{mT}$ in the absence of vortices in the disks. At the strongest absorption the vortices 
are excited resonantly. Different polarization patterns have different resonance frequencies [15] that are obtained from the FMR measurements. The patterns with a state formation frequency of $f_{\text {state }}=320 \mathrm{MHz}$ and $330 \mathrm{MHz}$ have comparable resonance frequencies of about $270 \mathrm{MHz}$, whereas the pattern with $f_{\text {state }}=270 \mathrm{MHz}$ has a resonance frequency of about $310 \mathrm{MHz}$ [Figs. 2(b) and 2(c)].

In the following we compare the measurements to calculations based on an extended Thiele model [15,21] that describes the vortex as a quasiparticle. The minimization of all micromagnetic energies is expressed by a force $\vec{F}=-\vec{\nabla} E$. The exchange and demagnetization energy are described by a two-dimensional harmonic potential $E_{\text {harm }}$. External magnetic fields are taken into account by a Zeeman term $E_{\text {zee }}$ [25]. The deflection of a vortex from its equilibrium position leads to magnetic surface charges and therefore to coupling between the disks. The interaction between neighboring vortices $j$ and $k$ is described via the magnetostatic energy between the side surfaces $E_{\mathrm{int}}=\xi C_{i} C_{j}\left(\hat{\eta}_{i j} \cdot \vec{X}_{j}\right) \cdot \vec{X}_{i}$ with a scaling factor $\xi<1$ [13], $\vec{X}_{i, j}$ the vortex core position vectors, and the interaction matrix $\hat{\eta}$ depending on the geometry, the magnetic material properties, and the relative position of the vortices $[12,13]$. The calculations are performed using the same spatial dimensions and number of disks of the crystal as in the experiments. A saturation magnetization of $M_{s}=800 \frac{\mathrm{kA}}{\mathrm{m}}$ and a resonance frequency of an isolated vortex of $\frac{\omega_{0}}{2 \pi}=300 \mathrm{MHz}$ for sample type I and $\frac{\omega_{0}}{2 \pi}=240 \mathrm{MHz}$ for sample type II are assumed. In the Thiele equations of motion damping is considered $[9,12,25]$, and a damping coefficient of $\Gamma=0.02 \cdot \omega_{0}$ according to Ref. [26] is used in the calculations. The steady state motions of every single vortex are calculated by the numerical solution of coupled Thiele equations [15]. A unidirectional oscillating magnetic field $\vec{H}_{\text {rf }}=\left(H_{0} \sin (\omega t), 0,0\right)$ excites all vortices. By using different frequencies the absorption spectra are calculated by means of the squared average velocities of the vortices that are proportional to the absorption in the FMR measurements [27]. The chiralities do not influence the coupling strength within the Thiele model. In the experiments they are randomly distributed.

In Fig. 2(c) the measured absorption is plotted together with Lorentzian fits for state formation frequencies of $270 \mathrm{MHz}$ (solid dark blue), $320 \mathrm{MHz}$ (dashed black), and $330 \mathrm{MHz}$ (dotted brown) versus the absorption frequency. In Fig. 2(d) the simulated absorptions for the three measured polarization patterns are depicted. The emergence of the polarization patterns can directly be substantiated by comparing the absorption peaks with the corresponding state formation frequencies. At low excitation frequencies the horizontally striped polarization pattern has the lowest absorption. It is stabilized at low state formation frequencies. In contrast the domains of homogeneous polarization are stabilized by high state formation frequencies. FMR spectroscopy and STXM experiments are in agreement with the calculations. We conclude that the observed states of homogeneous domains and horizontally striped polarization are reliably tuned.

\section{BAND STRUCTURE OF VORTEX CRYSTALS}

Having control over the two different polarization patterns, we will now determine their dispersion relations $\omega(k)$.

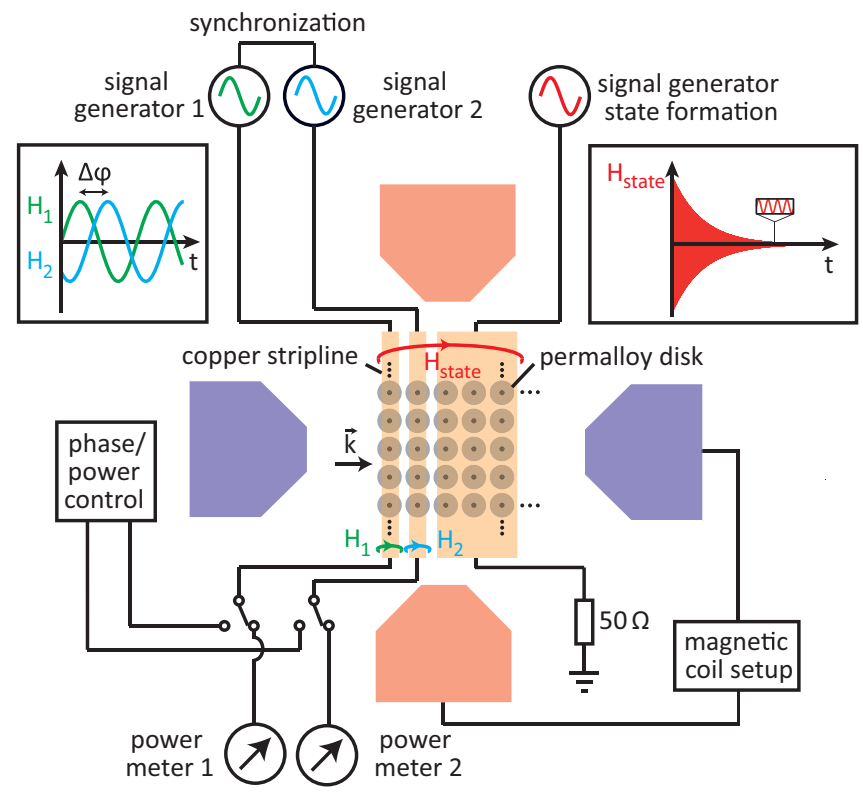

FIG. 3. (Color online) Schematic representation of the measurement setup. The polarization pattern is tuned via an adiabatic reduction of a high frequency magnetic field excitation. The first two columns of vortices are excited via the copper striplines with phase-shifted harmonic high frequency magnetic fields $\vec{H}_{1}$ and $\vec{H}_{2}$. Phase and amplitude of the excitation are controlled and adjusted with the signal that passed the sample. The power absorption is determined with the help of a reference measurement at $\mu_{0} H=60 \mathrm{mT}$.

Therefore the collective motions of the vortices in the crystal are excited and probed by the first two columns of vortices using the two thin striplines of sample type II depicted in Fig. 1. Harmonic excitations $\vec{H}_{1}=\left(H_{0} \sin (\omega t), 0,0\right)$ and $\vec{H}_{2}=$ $\left(H_{0} \sin (\omega t+\Delta \varphi), 0,0\right)$ are applied to the first and second column of the crystal with different phase relations $\Delta \varphi$ creating waves with different wavelengths. Here, the boundary condition of the enforced phase relation of the first two columns allows excitation of collective motions with defined wave numbers. The resonance frequency of the crystal changes for different phase relations of the harmonic excitation.

The setup for the band structure measurements is shown in Fig. 3. Subsequent to the polarization state formation the vortices under the two thin striplines are excited with phaseshifted harmonic excitation fields $\vec{H}_{1}$ and $\vec{H}_{2}$. The amplitude of the output signals depends on the phase and frequency of the input signal. The input power is controlled so as to pass the same power through the sample for all frequencies and phases.

Figure 4 depicts absorption spectra depending on the phase difference between the exciting magnetic fields for two polarization patterns. The blue and red lines represent the maxima of the absorption determined by Lorentzian fits. The two polarization patterns, i.e., horizontal stripes and large domains, feature different frequency offsets $\omega(\Delta \varphi=0)$ of their collective motions. By increasing the phase difference between the exciting magnetic fields $\vec{H}_{1}$ and $\vec{H}_{2}$ the resonance frequency increases. The variation of the absorption frequency of the striped pattern [Fig. 4(c)] is stronger than for the homogeneous domain pattern [Fig. 4(a)]. In other words, the bandwidth of the strongly interacting striped pattern is larger 

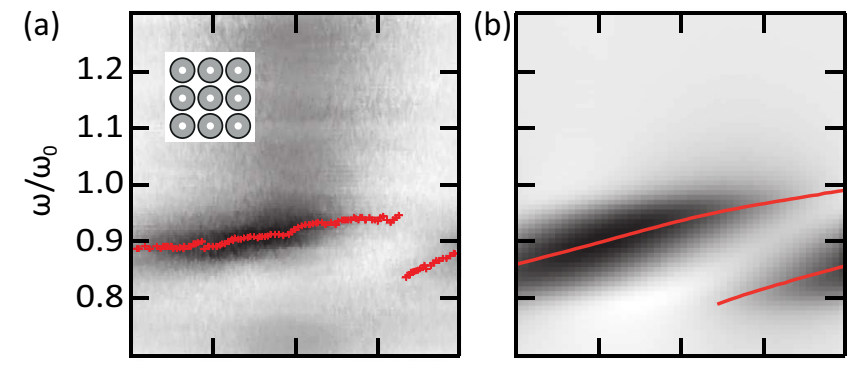

(c)

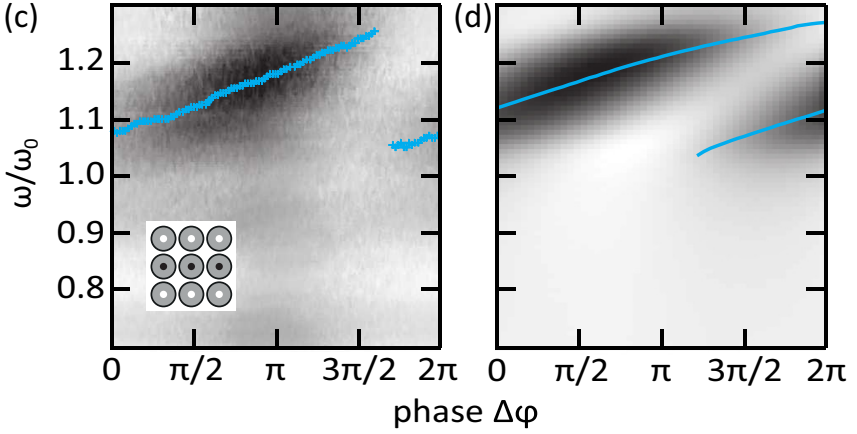

FIG. 4. (Color online) (a) Measured absorption intensity as a function of excitation frequency and phase difference between the fields $\vec{H}_{1}$ and $\vec{H}_{2}$ for domains of homogeneous polarization (inset) and (b) corresponding calculations based on the extended Thiele model, where $\frac{\omega_{0}}{2 \pi}=240 \mathrm{MHz}$. (c) Measured absorption intensity for horizontally striped polarization (inset) and (d) corresponding calculations. The red and blue markers and lines indicate the maxima of the absorption obtained from Lorentzian fits.

than the bandwidth of the weakly interacting homogeneous pattern. The band structure measurements are compared to calculations based on the extended Thiele model. The power absorption $P_{\text {abs }}$ under the first thin stripline is calculated numerically from the forces acting on the vortices over one oscillation period,

$$
P_{\mathrm{abs}}=\sum_{j=1}^{J} \frac{1}{\Delta t} \sum_{i=1}^{N} \int_{s_{i, j}\left(t_{j}\right)}^{s_{i, j+1}\left(t_{j+1}\right)} \vec{F}_{i} d \vec{s}_{i},
$$

where $J$ is the number of time steps $\Delta t$ during one oscillation period and $N$ is the number of vortices under the first stripline. The behavior of the whole crystal is taken into account by the coupling terms between the vortices. The calculated absorption agrees very well with the measurements as can be seen in Fig. 4. In the experiment only one resonance frequency is observed for each phase difference, whereas the calculations partly feature two absorption peaks. The absorption intensity of one peak in these phase regions is comparable to the signalto-noise ratio of the measurement setup. Small differences between experiment and calculations are explained by slight geometrical irregularities of the microdisks that lead to a decrease in bandwidth of the resonances.

The dispersion relation describing the frequency of waves dependent on their wave number $k$ [see coordinate system in Fig. 1(a)] is deduced from the relative phases of the exciting magnetic fields $\vec{H}_{1}$ and $\vec{H}_{2}$. In the Supplemental Material the propagation of waves through the crystal is experimentally shown for two phase differences $\Delta \varphi$ [23]. We observe that the imprinted phase difference results in different types of

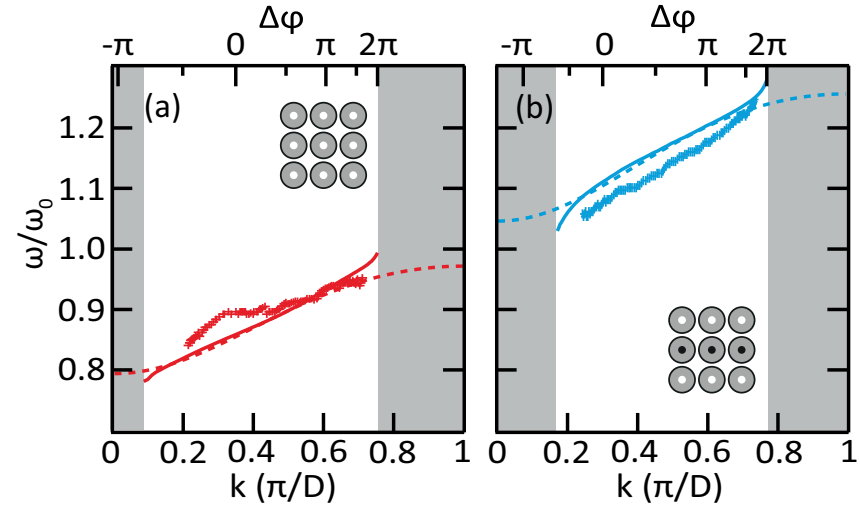

FIG. 5. (Color online) Band structure measurements (markers), calculations based on the extended Thiele model including damping (lines), and calculations by Shibata et al. [18] without damping (dashed lines). (a) Homogeneous polarization pattern. (b) Horizontally striped polarization pattern. The gray regions of wave numbers $k$ are experimentally not accessible. The resonance frequency of a single vortex is $\frac{\omega_{0}}{2 \pi}=240 \mathrm{MHz}$.

oscillations. The relative phases of the oscillations, illustrated by rotating arrows, are attributed to a specific wave number $k$. Here, a phase difference of $\Delta \varphi=92^{\circ}$ and $\Delta \varphi=174^{\circ}$ result in a wave number of $k=0.39 \pi / D$ and $k=0.66 \pi / D$, respectively. Thus, by increasing the phase difference $\Delta \varphi$ the wave number $k$ increases. We use numerical calculations to obtain the correlation between each phase difference $\Delta \varphi$ and wave number $k$. Comparable to the STXM measurements, the evaluation of the vortex core motions yields a wave number $k$ for every phase difference $\Delta \varphi$. Figure 5 combines the measured absorption (markers) with the calculations using the extended Thiele model (lines) and calculations for an infinite crystal without damping (dashed lines) obtained from Ref. [18]. The lower $x$ axis is the wave number $k$ obtained from the numerical calculations, while the upper $x$ axis shows the correlation to the phase difference $\Delta \varphi$. Wave numbers above and below a value of about $0.2 \pi / D$ and $0.8 \pi / D$ (gray regions) cannot be excited experimentally in the crystal. This is presumably caused by waves that are absorbed before being transmitted into the crystal due to low group velocities in the gap regions. This effect has also been observed for phononic crystals in the presence of damping [28]. The partial wave number gaps vanish for zero damping. The experimentally determined dispersion relations for domains of homogeneous polarization [Fig. 5(a)] and striped polarization [Fig. 5(b)] show the theoretically expected behavior. The band structure is reprogrammed by the polarization pattern tuning taking effect on the frequency offsets and the bandwidths of the dispersion relations.

\section{CONCLUSION}

In conclusion we have measured the dispersion relation of magnonic vortex crystals. The phase difference between the excitations has been translated into a wave number $k$, and the dispersion relation $\omega(k)$ is obtained. Two polarization patterns have been tuned: horizontal stripes and domains of homogeneous polarization. As the band structure depends on the polarization pattern, this is an example of band structure engineering via polarization tuning in vortex crystals. Our 
work enables studies of more complex systems with an increased number of bands using, for example, multivortex bases.

\section{ACKNOWLEDGMENTS}

We thank Ulrich Merkt for fruitful discussions and Michael Volkmann for superb technical assistance. We acknowledge the support of the Max-Planck-Institute for Intelligent Systems (formerly MPI for Metals Research), Department Schütz and the MAXYMUS team, particularly Michael Bechtel and Eberhard Goering. We thank the Helmholtz-Zentrum Berlin für Materialien und Energie for the allocation of synchrotron radiation beamtime. Financial support of the Deutsche Forschungsgemeinschaft via the Sonderforschungsbereich 668 and the Graduiertenkolleg 1286 is gratefully acknowledged. This work has been supported by the excellence cluster "The Hamburg Centre for Ultrafast Imaging - Structure, Dynamics, and the Centre of Matter at the Atomic Scale" of the Deutsche Forschungsgemeinschaft.
[1] H. Amano, N. Sawaki, I. Akasaki, and Y. Toyoda, Appl. Phys. Lett. 48, 353 (1986).

[2] S. Nakamura, T. Mukai, and M. Senoh, Appl. Phys. Lett. 64, 1687 (1994).

[3] V. V. Kruglyak, S. O. Demokritov, and D. Grundler, J. Phys. D: Appl. Phys. 43, 264001 (2010).

[4] B. Lenk, H. Ulrichs, F. Garbs, and M. Münzenberg, Phys. Rep. 507, 107 (2011).

[5] T. Shinjo, T. Okuno, R. Hassdorf, K. Shigeto, and T. Ono, Science 289, 930 (2000).

[6] A. Wachowiak, J. Wiebe, M. Bode, O. Pietzsch, M. Morgenstern, and R. Wiesendanger, Science 298, 577 (2002).

[7] B. Van Waeyenberge, A. Puzic, H. Stoll, K. W. Chou, T. Tyliszczak, R. Hertel, M. Fähnle, H. Brückl, K. Rott, G. Reiss, I. Neudecker, D. Weiss, C. H. Back, and G. Schütz, Nature (London) 444, 461 (2006).

[8] T. Kamionka, M. Martens, K. W. Chou, M. Curcic, A. Drews, G. Schütz, T. Tyliszczak, H. Stoll, B. Van Waeyenberge, and G. Meier, Phys. Rev. Lett. 105, 137204 (2010).

[9] K. Y. Guslienko, B. A. Ivanov, V. Novosad, Y. Otani, H. Shima, and K. Fukamichi, J. Appl. Phys. 91, 8037 (2002).

[10] S.-B. Choe, Y. Acremann, A. Scholl, A. Bauer, A. Doran, J. Stöhr, and H. A. Padmore, Science 304, 420 (2004).

[11] A. Vogel, A. Drews, T. Kamionka, M. Bolte, and G. Meier, Phys. Rev. Lett. 105, 037201 (2010).

[12] J. Shibata, K. Shigeto, and Y. Otani, Phys. Rev. B 67, 224404 (2003).

[13] C. F. Adolff, M. Hänze, A. Vogel, M. Weigand, M. Martens, and G. Meier, Phys. Rev. B 88, 224425 (2013).

[14] A. Vogel, M. Hänze, A. Drews, and G. Meier, Phys. Rev. B 89, 104403 (2014).
[15] M. Hänze, C. F. Adolff, M. Weigand, and G. Meier, Appl. Phys Lett. 104, 182405 (2014).

[16] M. Hänze, C. F. Adolff, M. Weigand, and G. Meier, Phys. Rev. B 91, 104428 (2015).

[17] D.-S. Han, A. Vogel, H. Jung, K.-S. Lee, M. Weigand, H. Stoll, G. Schütz, P. Fischer, G. Meier, and S.-K. Kim, Sci. Rep. 3, 2262 (2013).

[18] J. Shibata and Y. Otani, Phys. Rev. B 70, 012404 (2004).

[19] A. Y. Galkin, B. A. Ivanov, and C. E. Zaspel, Phys. Rev. B 74, 144419 (2006).

[20] O. V. Sukhostavets, J. González, and K. Y. Guslienko, Phys. Rev. B 87, 094402 (2013).

[21] A. A. Thiele, Phys. Rev. Lett. 30, 230 (1973).

[22] S. Jain, V. Novosad, F. Y. Fradin, J. E. Pearson, V. Tiberkevich, A. N. Slavin, and S. D. Bader, Nat. Commun. 3, 1330 (2012).

[23] See Supplemental Material http://link.aps.org/supplemental/ 10.1103/PhysRevB.91.224417 for movies and a detailed description of the two STXM measurement techniques.

[24] The polarization patterns for $f_{\text {state }}=270 \mathrm{MHz}$ and $330 \mathrm{MHz}$ are determined by out-of-plane measurements of the vortex magnetization while the polarization patterns for $f_{\text {state }}=320 \mathrm{MHz}$ are determined by in-plane contrast measurements.

[25] B. Krüger, A. Drews, M. Bolte, U. Merkt, D. Pfannkuche, and G. Meier, Phys. Rev. B 76, 224426 (2007).

[26] M. Martens, T. Kamionka, M. Weigand, H. Stoll, T. Tyliszczak, and G. Meier, Phys. Rev. B 87, 054426 (2013).

[27] A. Drews, B. Krüger, G. Selke, T. Kamionka, A. Vogel, M. Martens, U. Merkt, D. Möller, and G. Meier, Phys. Rev. B 85, 144417 (2012).

[28] M. I. Hussein and M. J. Frazier, J. Appl. Phys. 108, 093506 (2010). 